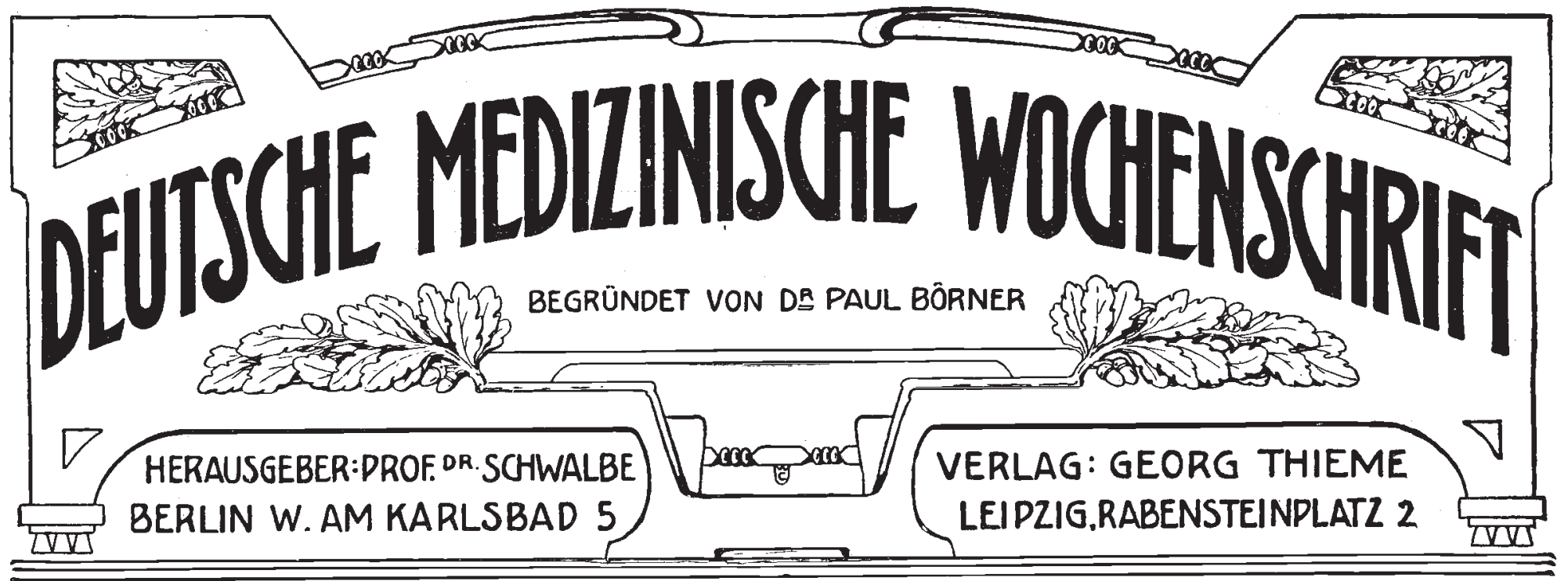

\section{Ueber Diagnose und Behandlung der Extrauteringravidität.}

Klinischer Vortrag

Von A. Martin in Berlin (-Greifswald).

(Fortsetzung aus No. 17.)

Nach meiner Auffassung sind wir nur unter besonders günstigen Umständen imstande, angesichts einer im schweren Kollaps infolge von Fruchtkapselaufbruch angetroffenen Frau mit hinreichender Sicherheit zu erkennen, ob es sich hier um einen tubaren Abort oder um eine Tubenruptur handelt. Selbst wenn der intraabdominale Blutverlust im Augenblick nicht so erheblich ist, daß das Leben zweifellos unmittelbar bedroht erscheint, so bleibt zu bedenken, daß auch bei dem inneren Fruchtkapselaufbruch Nachschübe der Blutung nicht nur in kurzer Frist, sondern auch nach Tagen und Wochen bei anscheinend geringfügigen Anstrengungen der Bauchpresse, bei einer Stuhlentleerung, beim Aufrichten im Bett, beim Erbrechen auftreten und nunmehr der Entkräfteten unmittelbar gefährlich werden können.

Anderseits ist die Sicherheit, daß das in die Bauchhöhle entleerte Fi und Blut in einer für die Frau lebenssicheren Weise zur Resorption gelangt, nicht so zuversichtlich gegeben, daß das Vertrauen darauf bei der Abwägung der Gefahr einer sofortigen Operation ausschlaggebend in die Wagschale für das Abwarten gelegt werden dïrfte.

Es ist nicht zu bestreiten, daß bei einer sonst Gesunden. unverkennbar Widerstandskräftigen ein nur leichter Kollapsanfall, bei dem der lokale Befund ohnehin begründete Zweifel an der Diagnose einer ektopischen Schwangerschaft. zuläßt, zunächst ein Abwarten gestattet. Aber auch hier weisen zahlreiche Beobachtungen darauf hin, da $\beta$ es dringend geboten ist, solche Frauen in bezug auf die ihnen drohenden Gefahren nicht zu täuschen. Solche Frauen gehören jedenfalls unter die stete Kontrolle eines operationskundigen Anstaltsleiters, in ein Krankenhaus oder Sanatorium, welches die Möglichkeit bietet, bei sachverständiger Beobachtung auf entsprechende Anzeichen hin sofort die blutstillende Operation auszuführen.

Anderseits können auch unmittelbar schwere Kollapsanfälle verhältnismäßig rasch, sozusagen vor unseren Augen, überwunden werden. Selbst wenn die Anämie stark ausgeprägt ist, beobachten wir, daß der Puls kräftiger wird, der Lufthunger nachläßt, ein behaglicher Schweiß sich einstellt. Es gelingt durch die Darreichung von Exzitantien per os oder anum, subkutan oder intravenös, durch reichliche Kochsalzinfusionen den Kräftezustand zu heben, auch behält der Magen andauernd entsprechende Stärkungsmittel und Nahrung. Dann ist man allerdings berechtigt zu warten, um zu versuchen, die Diagnose der ektopischen Schwangerschaft und der in ihr sich abspielenden Entwicklung zu sichern.
Dabei ist eine Klarstellung des Befundes am Leib und im Becken in erster Linie anzustreben.

Das sowohl beim inneren als auch besonders beim äußeren Aufbruch in die Bauchhöhle ergossene. Blut breitet sich in den meisten Fällen rasch durch die ganze Peritonealhöhle aus. Soweit es reicht, ist der Perkussionsschall absolut gedämpft - meist in scharfer Grenze, seltener mit einer deutlich nachweisbaren schmalen Zone helleren Schalles. Dabei ist der Leib meist ausgesprochen empfindlich gegen Berührung, oft sind die Bauchdecken. hart gespannt. Früh stellt sich deutlicher Meteorismus ein.

Schon nach Stunden gerinnt das Blut teilweise: nach 24 bis 30 Stunden werden Coagula im Becken wahrnehmbar. Das hintere Scheidengewölbe ist herabgedrängt, der Uterus nach vorn, meist etwas seitlich verlagert, wenn er nicht in Retroversio-flexio liegt und im ganzen herabgedrängt wird, während das Gerinnsel die Excavatio vesico-uterina füllt und das vordere Scheidengewölbe gespannt erscheinen läßt. Gelingt es, bimanuell den Beckeninhalt zu prïfen, so imponiert die Seite des Eisitzes durch eine unbestimmte, aber immerhin hinreichend deutlich wahrnehmbare, teigig-weiche Massenausfüllung. Erst nach weiteren 8-12 Stunden, wenn der Gerinnungsprozeß vorgeschritten ist, wird die Masse deutlicher fühlbar. Zuweilen gelingt es, von der Uteruskante aus die Tube zu fühlen, vergrößert, weich. Zuweilen gelingt es auch, in dem Ganzen eine etwas derbere Masse von Eigröße und je nachdem von größerem Umfang, die Frucht, die Fruchthüllen mit dem Foetus darin in dem Blutklumpen zu unterscheiden. Da man aber stets der Gefahr eingedenk sein muß, daß nuan durch intensiveres Tasten neue Störungen in Fruchthalter verursacht, muß man sich mit einer approximativen Feststellung begnügen.

Nur ausnahmsweise gelingt es, die vergrößerte, weiche Tube mit voller Deutlichkeit herauszutasten, ev. auch noch daneben einen weichen Klumpen des Eis. Dann dürfte allerdings die Diagnose des tubaren Abortes mit Zuversicht gestellt werden

Weitaus häufiger ist eine solche Sicherheit nicht zu erlangen. Bei der Undurchsichtigkeit des Befundes muß die Diagnose in der Vorgeschichte und in dem Vorgang der Katastrophe selbst ihre Ergänzung finden. Angesichts der gefahrdrohenden Sachlage zu warten, bis der weitere Verlauf entscheiden läßt, ob ein Abort oder eine Ruptur erfolgt ist, wird nur dann erlaubt sein, wenn alsbald nach Ankunft des Arztes die Shockwirkung ihr bedrohliches Ansehen verliert, der Puls kräftiger und gleichmäßiger wird, die Atmung zur Norm zurückkehrt, die Nahrungsaufnahme nicht durch Erbrechen gestört wird, keine abundante Genitalblutung auftritt.

Die Entscheidung über den Vorgang selbst ist besonders dadurch erschwert, daß der Fruchtkapselaufbruch sich nicht immer in einem Akt vollzieht, daß längere und kürzere Intervalle sowohl beim Abort als bei der Ruptur nicht selten sind. Weiter kommt in Betracht, daß beide Arten des Aufbruchs gleichzeitig an derselben schwangeren Tube zur Entwicklung kommen. 
Eine weitere Schwierigkeit bildet die Beobachtung, daß bei den meisten ektopischen Schwangerschaiten ausgedehnte Verwachsungen, besonders mit der durch chronische Salpingitis der anderen Seite und Perimetritis verunstalteten und verlagerten Tube die gesamten Beckenorgane untereinander verbacken. Entzündungsprozesse der Genitalien, Neubildungen am Uterus und den Ovarien verursachen eine oft unentwirrbare Verschleierung des Befundes.

In dem letzten Jahrzehnt haben sich bemerkenswerterweise die Beobachtungen von gleichzeitiger intra- und extrauteriner Schwangerschaft vermehrt: jedenfalls zwingen solche Beobachtungen, eine derartige Komplikation heute stets in den Bereich der Möglichkeiten zu stellen und dementsprechend bei der Untersuchung zu berüicksichtigen. Der schwangere Uterus sollte mit seiner Dehnung des Corpus, der Konsistenzveränderung, den Konsistenzschwankungen durch Kontraktionen und seiner Reaktion auf den Reiz der Palpation gegenüber dem danebenliegenden Tubensack eigentlich deutlich erkennbar sein.' Aber ganz abgesehen von der gebotenen Vorsicht bei der Abtastung wird die Entscheidung durch die Inkongruenz des Verlaufes dieser Art von Zwillingseiern erheblich erschwert. Das eine Ei wird durchblutet, stirbt ab, während das andere nicht gleichzeitig, wenn überhaupt in seinem Fruchthalter getroffen wird. Der Vorgang des uterinen Abortes mit dem erheblichen Blutverlust, der Beruhigung des Zustandes in dem Augenblick der Entleerung des Eis tritt ganz in den Vordergrund; dann läßt der Kollaps nach, er läßt die Ueberwindung des Höhepunktes der Gefahr hoffen. Der geübte Untersucher findet dann wohl heraus, daß neben dem entleerten massigen Uteruskörper noch eine weiche Masse liegt, welche annähernd einem dem eben entleerten Ei gleichaltrigen ektopischen entspricht. - Man hat auf die Spindelform der schwangeren Tube Gewicht gelegt. Zuweilen trifft das zu, häufiger nicht. Gelingt es dem geübten Untersucher, vom Uterushorn aus die Ausbuchtung der Tube festzustellen, deren Weichheit mit der lebhaften Pulsation der Blutgefäße in und unter derselben in der Tat sehr prägnant zur Wahrnehmung kommen kann, dann wird die Diagnose wohl wesentlich gegeben erscheinen. Schwieriger jedenfalls wird die Klarstellung, wenn die Katastrophe zuerst im ektopischen Fruchtsacke auftritt. Im allgemeinen scheint diese Art der Aufeinanderfolge die häufigere zu sein, da die Durchwachsung der Wand des ohnehin kaum zulänglichen Fruchthalters und die Störung der plazentaren Verhältnisse von vornherein zunächst für die Katastrophe in der Tube prädisponieren. Die Diagnose stößt auf die größten Schwierigkeiten, wenn der Uterus schon durch seine Ausdehnung die genaue Abtastung erschwert und eine solche durch die Rücksicht auf die Hervorrufung einer Schwangerschaftunterbrechung kompliziert ist.

Auf der Höhe des Anfalls ist es auBerordentlich schwer zu entscheiden, ob es sich um einen inneren oder äuBeren Fruchtkapselaufbruch handelt, ob Abort oder Ruptur vorliegt. Erholt sich die Patientin bald und gelingt es, den zusammengefallenen Tubensack, das Ei und das geronnene Blut nachzuweisen, dann ist Abort als das Wahrscheinlichste anzusehen. Ist die Patientin unter sachverständiger Kontrolle - Ueberführung in ein Hospital mit sachkundigem Leiter — so darf man abwarten, muß aber stets zum Eingriff bereit sein, denn auch die Ausstoßung des Eis aus dem Ostium tubae abdominale vollzieht sich nicht selten absatzweise unter wiederholter und deletärer Blutung: es kann währenddessen auch noch eine Ruptur des Fruchthalters mit allen ihren Folgen eintreten!

Erholt sich die Patientin nicht schnell in unverkennbarer Weise, so ist andauerndes Bluten der angerissenen Tubenwandgefäße um die Plazentastelle anzunehmen, die Folge einer Ruptur. Dann erscheint die unmittelbare Blutstillung geboten.

Ist eine durchsichtige Vorgeschichte gegeben, so wird man auf die Diagnose einer Extrauterinschwangerschaft überhaupt, und wenn eine Katastrophe erfolgt ist, auf Fruchtkapselaufbruch mit ziemlicher Sicherheit schließen dürfen. Analoge Katastrophen sieht man bei akuter Peritonitis, wenn eine eitrige Salpingitis ihre Infektionsträger (Gonokokken, Streptokokken) aus dem Ostium abdominale austreten läßt. Auch im Verlaufe einer puerperalen Infektion kommt es in ähnlicher Weise zu akuter Exazerbation des gefahrvollen Zustandes, wenn das Peritoneum plötzlich ergriffen wird.

Im Anfall selbst kann es schwer sein, diese Vorgänge voneinander $\mathrm{zu}$ unterscheiden. Allerdings sind hier in der Regel prägnante, von den Kranken selbst und ihrer Umgebung bemerkte Beschwerden vorausgegangen. Die Kranken waren schon einige Zeit in ärztlicher Beobachtung. Die Spuren der Erkrankung sind unverkennbar aufzufinden: Gonokokken, Streptokokken. Besonders bedeutungsvoll ist es, wenn die Patientinnen schon vorher gefiebert haben und mehr oder weniger über heftige Schmerzen im Schoß klagten. Die Katastrophe selbst verläuft dann unter Schüttelfrost, jähem Temperaturanstieg, plötzlicher und langdauernder Schmerzsteigerung, Symptome, welche gerade bei Fruchtkapselaufbruch prägnant nur während des Abortus oder der Ruptur andauern. Fieber begleitet diesen Vorgang nur selten; es entwickelt sich erst, wenn das Peritoneum auf den Vorgang in der Tube und die dabei austretenden reizenden Gebilde (Blut-Ei) reagiert, besonders aber wenn hierbei auch infektiöses Material aus der Tube verschleppt wird.

In gleicher Weise erleichtert die Unterscheidung in der Regel die Vorgeschichte einer Perityphlitis, einer Cholecystitis, der Darmperforation und ähnlicher Erkrankungen. Diese sind zudem in der großen Mehrzahl der Fälle mehr oder weniger typisch lokalisiert. Bei ihnen setzt die Katastrophe unter den prägnanten Symptomen der akuten Peritonitis mit rasch markiertem Kräfteverfall ein.

Die Therapie im akuten Stadium des Fruchtkapselaufbruches des tubaren Abortus und der tubaren Ruptur besteht für den in diesem Stadium bedrohlicher Lebensgefahr hinzutretenden Arzt in der tunlichst unmittelbaren Sicherung gegen weitere intraabdominale Blutverluste, also in sofortiger Operation. Auffallenderweise vertragen solche Kranken im Kollaps die Erschütterung der Ueberführung in Krankenhäuser, ja auch stundenlange Wagenfahrten überraschend gut. Freilich sieht man vereinzelt auch, daß sie auf dem Wege selbst erliegen oder in Agone ankommen. Wenn also die Asepsis hinreichend gewährleistet ist, dann ist die Operation am Orte selbst, also im Privathause, vorzuziehen. Andernfalls muß die Aussicht, daß im Krankenhaus der Patientin alle Wohltaten vollendeter Asepsis zugute kommen, die Bedenken gegen den Transport zurückdrängen.

Immer wieder erheben sich Stimmen zugunsten wenigstens eines kürzeren Abwartens, um die Operation nicht auf der Höhe des Kollapses vornehmen zu müssen. Meine Erfahrungen lassen mich dem bestimmt widersprechen. Die Operation, in schnellen Zügen ausgeführs, bildet an sich keine nennenswerte Gefahr. Die hochgradige Anämie erübrigt oft die Notwendigkeit einer Narkose, dit sich auf den Aetherrausch oder eine lokale Anästhesie während der Spaltung der gewaschenen und mit Jodtinktur bestrichenen Bauchhaut beschränken kann. Fin entschlossener Griff fördert die blutende Gewebsmasse, die Tube, soweit zutage, daß sie eventuell zunächst mit Arterienklemmen versorgt werden kann: danit ist re vera die Operation erledigt. Oft bessert sich fast in demselben Augenblick die Pulsqualität. Fast unblutig kann der Fruchthalter völlig entleert und entfernt werden. Die Versorgung des in Klemmen gefaßten Stieles kann ohne weiteren Blutverlust erfolgen, ebenso die Peritonealisierung der Schnittfläche, die Reinigung der Bauchhöhle und der Verschluß der Bauchwunde. Inzwischen können alle Mittel zur Bekämpfung der Anämie ungestört zur Anwendung kommen. Vor der provisorischen Blutstillung an der Tube ist von der Einführung physiologischer Kochsalzlösung abzustehen.

Etwaigen Komplikationen zu begegnen, z. B. die Entbindung des gleichzeitig schwangeren Uterus, die Ausräumung der Decidua uterina vorzunehmen, wenn diese unter Blutabgang zur Abstoßung kommt - das sind Aufgaben, deren Erledigung (Ausräumung des Cavum und Sorge für eine gründliche Kontraktion des Uterus) sich im unmittelbaren Anschluß in der Regel ohne Schwierigkeiten von selbst ergibt. 
B. Das subakute und das chronische Stadium. (Haematocele intraperitonealis.)

Wenn nach unserer heutigen Erfahrung ein sehr großer Teil der Fälle von ektopischer Schwangerschaft sicherlich in dem akuten Stadium des Fruchtkapselaufbruches zur Beobachtung kommt, so stellt sich doch eine nicht kleine Reihe erst in einem mehr chronischen Zustand zur Erkenntnis und Behandlung. Nicht mehr die akute Anämie und die so bedrohlichen Begleiterscheinungen der Katastrophe selbst führen uns an das Krankenbett, sondern der mehr oder weniger stark ausgesprochene tiefe Leidenszustand, welchen das aus seinem Fruchthalter teilweise oder vollständig ausgestoßene $\mathrm{Ei}$ und das dabei ergossene Blut verursacht, weil die Aufgabe seiner Resorption und Organisation die Leistungsfähigkeit der Frau übersteigt, oder weil ein infektiöser Vorgang diese kompliziert.

Um die Aufgaben der Diagnose und der Therapie klarzulegen, müssen wir uns auch hier die Möglichkeiten des Krankheitsverlaufes vergegenwärtigen.

Das Ei kann bei dem inneren Fruchtkapselaufbruch, bei dem Tubarabort - der zugleich durch Eindringen von Blut zwischen die Eihäute und in den amniotischen Sack dasselbe zerstört, sodaß Foetus und Fruchtwasser daraus entleert werden - in der Ampulla tubae liegen bleiben oder in die Bauchhöhle gelangen. Hier gerät es zwischen die Pseudomembranen, welche die Beckenorgane in solchen Fällen in großer Häufigkeit umspinnen, oder es gelangt zwischen die Därme. In der Regel wird dabei die Verbindung des Foetus mit dem Ei vernichtet, soda $B$ beide 'Teile für sich der resorbierenden. Kraft des Peritoneum ausgesetzt werden. Das bei dem Aufbruch entleerte Blut wird zunächst durch den ganzen Bauchraum verbreitet. Kommt es zur Gerinnung, so lagern. die Massen nach dem Gesetz der Schwere in der Excavatio recto-uterina, aber auch zwischen den Pseudomembranen in allen Teilen des kleinen Beckens, in der ganzen. Bauchhöhle. Der flüssige Teil kann in einigen Tagen resorbiert werden. Die Gerinnsel kapseln sich ein (Haematocele intraperitonealis). In der langsam an Dicke zunehmenden derben äußeren Schale bleibt in Innern eine dickflüssige Masse, welche noch monatelang der Gerinnung widersteht. Diese harte Schale verhindert oft lange Monate hindurch die Resorption. Dabei hinterbleibt die innige Verlötung der Außenfläche dieser einen förmlichen Tumor darstellenden Kapsel-Hä matocele mit der Nachbarschaft.

Im weiteren Verlauf kann das aus dem Ostium tubae abdominale entleerte Ei zum Lithopädion werden, mit Verkalkung der Eischale, der Plazenta, zuletzt auch des Foetus. Viel häufiger wird der Foetus nach und nach bis auf die verknöcherten Skeletteile, die schließlich aus allen Verbindungen gelöst sind, resorbiert.

Gewöhnlich übt das Blut mit dem Ei einen starken Reiz auf das Peritoneum aus: die Zeichen seiner Reaktion sind ausgedehnte fibröse Ablagerungen und die daraus entwickelten Verklebungen aller Bauchorgane untereinander und mit dem Bauchfell.

Der äuBere Fruchtkapselaufbruch (Ruptura tubae) fügt diesen Befunden in verschiedenen Richtungen Varianten hinzu. Das Ei, besonders die Plazenta, kann in beschränkter oder weiterer Ausdehnung mit der Basis in Verbindung bleiben: der Foetus wird durch die unverletzte Nabelschnur auch bei extramembranöser Lagerung weiter ernährt. Der Fruchtkapselaufbruch führt also nicht zu einer Unterbrechung der Schwangerschaft.

Eine andere bedeutungsvclle Variante bilden die Einbrüche zwischen die Blätter des Ligamentum latum, sodaß das Blut vollständig oder teilweise zunächst wenigstens außerhalb der Peritonealhc̈hle liegt, mit dem Ei oder Teilen desselben (Haematoma extraperitoneale). Bei größerer Massigkeit des Blutergusses kommt es in der Regel nach und nach doch zum Einbruch in das Cavum peritonei, ebenso bei weiter vorgeschrittener Eiausbildung und größerer Gewalt des Aufbruches, mit und ohne Mitwirkung eines veranlassenden Traumas.

In allen solchen Fällen ist Resorption und Heilung möglich, aber es bedarf dazu in der Regel langer Zeit, in welcher alle möglichen Störungen zu gewärtigen sind. Unter diesen spielt die Beteiligung anderer Organe, besonders von Blase und Darm, eine große Rolle. Noch verhängnisvoller wird die Infektion, sei es durch vorher in den Genitalien (Streptokokken, Staphylokokken, Gonokokken), im Darm (Appendix-Bacterium coli) oder anderen Organen domizilierende Keime oder durch solche, welche im Laufe der Heilung, besonders bei Gelegenheit therapeutischer Hilfsversuche, eingebracht werden. Dann bieten Blut und $\mathrm{Ei}$, das durch diese gereizte Peritoneum, die Wunde in der Tube den allzu bereiten Nährboden. Es kommt zur AbszeBbildung mit der naheliegenden Gefahr einer raschen Mitbeteiligung des Peritoneum und aller Nachbarorgane. Auch dann kann die Virulenz der Eitererreger frühzeitig erlöschen, unter Resorption des Eiters auch Lithopädionbildung und Heilung eintreten. Viel häufiger kommt es schnell zum Durchbruch und zur Entleerung nach außen durch die nächstbeteiligten Organe oder unter Einschmelzung z. B. der Bauchdecken. Dabei wird endlich auch das Ei entleert, dessen einzelne Teile völlig eingeschmolzen sind. Häufiger erliegt die Frau, deren Widerstandskraft schon durch die Katastrophe des Fruchtkapselaufbruches gebrochen war. (Schluß folgt.) 